\title{
Cardiac damage and dysfunction in diabetic cardiomyopathy are ameliorated by Grx1
}

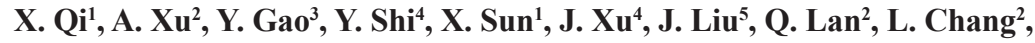 \\ C. Zhang ${ }^{4}$ and $\mathrm{H}$. $\mathbf{Y u}^{6}$
}

${ }^{1}$ Department of Clinical Biochemistry, Qiqihar Medical University, Heilongjiang, Qiqihar, China

${ }^{2}$ Undergraduate Program of Qiqihar Medical University, Heilongjiang, Qiqihar, China

${ }^{3}$ Clinical Laboratory of Third Affiliated Hospital, Qiqihar Medical University, Heilongjiang, Qiqihar, China ${ }^{4}$ Department of Biochemistry, Qiqihar Medical University, Heilongjiang, Qiqihar, China

${ }^{5}$ First Affiliated Hospital, Qiqihar Medical University, Heilongjiang, Qiqihar, China

${ }^{6}$ Department of Biology Genetics, Qiqihar Medical University, Heilongjiang, Qiqihar, China

Corresponding authors: C. Zhang / H. Yu

E-mail: cjzhang2005@163.com / yht422@126.com

Genet. Mol. Res. 15 (3): gmr.15039000

Received July 21, 2016

Accepted August 1, 2016

Published September 19, 2016

DOI http://dx.doi.org/10.4238/gmr.15039000

Copyright $(2016$ The Authors. This is an open-access article distributed under the terms of the Creative Commons Attribution ShareAlike (CC BY-SA) 4.0 License

\begin{abstract}
Glutaredoxin 1 (Grx1) has been found to be an important endogenous antioxidant enzyme closely related to the pathogenesis of diabetes and cardiovascular diseases caused by oxidative stress. In this study, the functional changes of the Grx1 redox system in blood of hyperglycemic patients were examined. Furthermore, using a rat model of streptozotocin (STZ)- and high-fat-diet-induced type 2 diabetes,
\end{abstract}


we explored the correlation between functional changes of the Grx 1 redox system in the left ventricular tissue and blood of the diabetic rats. Moreover, we studied the protective effect of Grx1 against cardiac toxicity caused by the high-glucose-induced expression of cardiac matrix metalloproteinases (MMPs) in primary cultured cardiac fibroblasts. Finally, we investigated the protective effects and signaling regulatory mechanism of Grx1 against diabetic cardiomyopathy (DCM) in terms of oxidative stress and NF- $\mathrm{kB}$-mediated fibrosis-associated signaling pathways. In the serum of hyperglycemic patients, Grx1 levels were elevated, total/protein thiol or sulfhydryl (Total-SH/P-SH) levels were decreased, glutathione was downregulated, and oxidized glutathione was upregulated. In addition, in the left ventricular myocardium and blood of the diabetic rats, Grx1 levels were significantly increased and glutathione reductase and P-SH levels were decreased. Moreover, endogenous Grx1 was highly expressed in cardiac fibroblasts during high-glucose treatment, and exogenous Grx1 can prevent DCM by controlling oxidative damage and MMP expression. These findings are suggestive of changes in the Grx1 redox system, and Grx1-regulated protein oxidative modifications may serve as molecular markers for diabetes caused by high-glucose-induced oxidative stress.

Key words: Glutaredoxin 1; Diabetic cardiomyopathy; Oxidative damage; Cardiac fibrosis

\section{INTRODUCTION}

Diabetes is a serious disease that significantly worsens quality of life. The fundamental harm associated with diabetes lies in its various complications that can affect all major body organs, especially the cardiovascular system. Epidemiological and clinical data show that diabetes can significantly increase the probability of cardiac dysfunction. Diabetic cardiomyopathy (DCM) is a common chronic complication of diabetes. The main pathological manifestations of DCM include cardiac hypertrophy, increased ventricular weight/body weight ratio (cardiac mass index), focal myocardial necrosis, extracellular matrix (ECM) deposition, and diabetic myocardial fibrosis (DMF), which all severely endanger the patient's health. Thus, it is important to understand the pathogenesis of DCM and to explore new methods of prevention and treatment. DCM involves a complex pathological process, and its pathogenesis has not yet been fully elucidated. However, it is known that lipid disorders, insulin resistance, oxidative stress, chronic inflammation, apoptosis, microvascular disease, and DMF all play a role in the onset and development of DCM (Marine et al., 2013; Miki et al., 2013).

The clinical characteristic of diabetes is hyperglycemia. High blood glucose is a key initiating factor of DCM. As an independent risk factor, hyperglycemia can directly damage the myocardium, while the pathophysiological changes such as myocardial oxidative stress, inflammation, and fibrosis, on the basis of hyperglycemia, promote the progression of DCM. Studies have shown that reactive oxygen species (ROS) are involved

Genetics and Molecular Research 15 (3): gmr.15039000 
in the pathogenesis of DCM, and the presence of oxidative stress markers has been detected in the blood and myocardium of the patients. Oxidative stress and apoptosis of cardiomyocytes are molecular events of the early stage of DCM. Because of oxidative stress, myocardial structures change, and myocardial cells undergo apoptosis and are lost and replaced by collagen fibers. The surviving myocardium cannot compensate for these losses effectively, and therefore heart failure ensues, which is a significant factor in cardiac dysfunction at the late stages of DCM.

High-glucose-induced oxidative stress is a key pathological factor that induces DMF (Aragno et al., 2008). The most common and serious complication of diabetes is excessive accumulation of connective tissue (pathological fibrosis) (Ernande and Derumeaux, 2012; Li et al., 2012). DMF occurs mainly at the late stage of DCM, whereas the symptoms of the early stage of DCM mainly include myocardial dysfunction and cardiac hypertrophy (Jellis et al., 2011). One factor that plays a central role in DCM is myocardial interstitial fibrosis. Excessive collagen deposition causes increased stiffness of the ventricular wall and reduced ventricular compliance, resulting in the systolic and diastolic dysfunction of the ventricle. Fortunately, active interventions can effectively slow the progression of DCM, postpone the onset of DMF, and reduce the morbidity and mortality of diabetic cardiovascular diseases. The exact molecular mechanism that triggers DMF has not yet been identified; this situation makes treatment a daunting task. Research into the mechanisms related to DMF is crucial for exploration of therapeutic targets as well as for prevention and postponement. Studies have confirmed that alleviation of oxidative stress is important for successful management of DMF (Li et al., 2012).

Human glutaredoxin 1 (Grx1) performs a wide range of antioxidant, anti-inflammatory, and antiapoptotic functions in the body. It is also involved in the regulation of various cellular functions, maintains a stable cellular redox state, produces a reducing environment within the cell under varying environmental conditions, and is involved in the pathogenesis of many diseases including diabetes and heart disease (Lekli et al., 2010; Chen et al., 2013a; De Benedetto et al., 2014; Du et al., 2014). In a physiological state of the cytoplasm, NADPH, GSH, GR, and Grx1 constitute the Grx1redox system, which reduces the oxidation state of proteinglutathione mixed disulfides (PSSG) in vivo and regulates post-translational modifications of thiol-containing proteins. Due to accumulation of GSSG and a lack of GSH, oxidative stress or a lack of energy can promote the oxidation state of the Grx1 redox system (Allen and Mieyal, 2012; Ghezzi, 2013). It was discovered that exogenous Grx1 has a significant protective effect against high-glucose-induced oxidative stress and apoptosis in the myocardium and vascular endothelial cells (Li et al., 2014; Zhang et al., 2014a,b). Nonetheless, the mechanism underlying the Grx1-driven regulation and improvement of cardiac function in diabetes has yet to be explained. Our aim was to clarify the impact of inhibition of hyperglycemia by Grx 1 on myocardial tissue and cells and its underlying mechanism. For this purpose, we used the blood of hyperglycemic patients, myocardial fibroblasts cultured under high-glucose conditions, and an animal model of streptozotocin (STZ)-induced type 2 diabetes to study the characteristics of myocardial damage caused by redox homeostatic changes during high-glucose treatment. In addition, we determined whether there is a protective effect against DCM due to functional changes in the endogenous Grx1 redox system or due to an intervention via exogenous Grx1. The goal was to clarify the molecular mechanisms of the protective effect of Grx1 against DCM in order to develop a novel method and theoretical basis for the prevention and treatment of DCM.

Genetics and Molecular Research 15 (3): gmr.15039000 


\section{MATERIAL AND METHODS}

\section{Materials}

Grx1 was purchased from ProSpec, Israel; carnosine was acquired from Sigma, USA; the ELISA kit for human and rat Grx1 determination was from Shanghai Wu Hao Economic, Ltd. The Redox Western blot kit (S7150) was purchased from Millipore; the antibodies against Grx1, GR, MMP-2, MMP-9, and p-NF- $\kappa B$ were acquired from Abcam, UK, and ECL Luminous Fluid from Thermo, USA. The T-GSH/GSSG test kit, glutathione S-transferase (GST), and total sulfhydryl (-SH) test kit were purchased from Nanjing Jiancheng, China, and the Mini RNA Isolation kit ${ }^{\mathrm{TM}}$ (R1030) and Titan TM One-Tube RT-PCR System from Roche.

\section{Quantification of clinical indicators}

We analyzed hyperglycemic patients with a new diagnosis (but not yet treated) in an endocrinology department of any affiliated hospital between October 2012 and August 2014. During the same period, the control group was assembled from healthy people of comparable age and gender after health screening. ELISA kits were used to determine the serum Grx1 and IL-22 levels. Commercial kits were used to determine the levels of GSH, GSSG, Total-SH, NO, and GST activity. Specific experimental procedures were conducted in strict accordance with the kit instructions.

\section{Determination of total concentrations of myocardial collagen, inflammatory cytokines, and Grx1/GR protein}

A rat model of type 2 diabetes for research on diabetic cardiovascular diseases was established. One week after initiation of the model, blood was collected for quantification of the indicators under study. The diabetic animal model group showed significantly increased blood glucose, triglycerides, cholesterol, and LDL, all at $\mathrm{P}<0.05$. In addition, the model group also demonstrated reduced insulin levels and thus insulin resistance according to the results of a radioimmunoassay with an r-911 automatic RIA counter. The blood glucose level was greater than $400 \mathrm{mg} / \mathrm{dL}$. At 3 and 6 months after initiation of the experimental diabetes, the heart tissues of the rats were collected for analysis of the indicators of myocardial fibrosis, inflammation, and oxidative damage using western blotting, RT-PCR, and histopathological methods. The myocardial hydroxyl acid level was used to assess the total amount of myocardial collagen. A portion of the left ventricular myocardium was fixed in liquid nitrogen for a myocardial hydroxyproline assay. The chloramine $\mathrm{T}$ method was used to determine the hydroxyproline content, which was converted into a hydroxyproline amount per gram of myocardial tissue and then multiplied by 7.46 to obtain the total amount of myocardial collagen. Another part of the heart tissue was fixed in a $4 \%$ neutral formaldehyde solution for quantification of Grx1/GR protein by immunohistochemical staining. After total RNA was extracted from the rat cardiac muscle, RT-PCR was used to detect the expression of proinflammatory cytokines TNF- $\alpha$, IL-1B, and NF- $\kappa B$ p65. An ELISA kit was used to determine the blood Grx1 level, and the OxyBlot method was applied to quantification of the myocardial oxidative damage in the diabetic rats. A commercial kit was used to determine the oxidation degree of protein thiol groups (P-SH).

Genetics and Molecular Research 15 (3): gmr.15039000 


\section{Cell culture, RT-PCR and western blots}

The cardiac myocytes and primary cultured rat fibroblasts were cultivated in the DMEM medium with $10 \%$ of fetal bovine serum at $37^{\circ} \mathrm{C}$ and $5 \%$ of $\mathrm{CO}_{2}$. The experimental groups included the following: normal glucose (Glu) group (NC group, Glu concentration was $5.5 \mathrm{mM}$ ), high-glucose group (HG group, Glu concentration was $25 \mathrm{mM}$ ), Grx1 preprotection group (Grx1 + HG group, Grx 1 was added to the final concentration of $5 \mu \mathrm{g} / \mathrm{mL} 6 \mathrm{~h}$ in advance), positive control carnosine (Car) preprotection group (Car $+\mathrm{HG}$ group, Car was added to a final concentration of $20 \mathrm{mM} 6 \mathrm{~h}$ in advance), and Grx1 inhibitor cadmium $\left(\mathrm{Cd}^{2+}\right)$ group $\left(\mathrm{Cd}^{2+}\right.$ $+\mathrm{HG}$ group, $\mathrm{CdCl}_{2}$ was added to the final concentration of $10 \mu \mathrm{M} 6 \mathrm{~h}$ beforehand).

Cells were collected and lysed to extract proteins. The same amounts of protein samples were analyzed by SDS-PAGE. After the electrophoresis, proteins were transferred to a polyvinylidene difluoride (PVDF) membrane, which was later blocked with 5\% nonfat dry milk, incubated with anti-Grx, anti-MMP-2, and anti-MMP-9 antibodies and a horseradish peroxidase (HRP)-labeled antibody, and the resulting signals were detected by means of the ECLplus kit. Meanwhile, Actin/GAPDH served as loading controls. A fluorescence and chemiluminescence imaging system (ChemiDocTMXRS, Bio-Rad) was employed to capture western blot images.

Polymerase chain reaction (PCR) conditions included the following: initial denaturation at $94^{\circ} \mathrm{C}$ for $5 \mathrm{~min}$; denaturation at $94^{\circ}-95^{\circ} \mathrm{C}$ for $30 \mathrm{~s}$, annealing at $55^{\circ} \mathrm{C}$ for $30 \mathrm{~s}$, extension at $72^{\circ} \mathrm{C}$ for $30 \mathrm{~s}$ for a total of $25-28$ cycles; then final extension at $72^{\circ} \mathrm{C}$ for $10 \mathrm{~min}$ and holding at $4^{\circ} \mathrm{C}$. Agarose gel electrophoresis was used to analyze PCR products, and the images were captured by means of the gel imaging system. The results were analyzed in the Quantity One software. The integral optical density (IOD) value of each band was read, and the expression levels of molecules from inflammatory pathways were analyzed according to the IOD ratios of NF- $\mathrm{KB}, \mathrm{TNF}-\alpha$, and $\beta$-actin bands.

\section{Statistical analysis}

All quantitative data were presented as mean \pm SD and analyzed with the GraphPad Prism software, version 5.0. Student $t$-test was used to compare two group means.

\section{RESULTS}

\section{Altered functions of the blood Grx1 redox system in diabetic patients}

High-glucose-induced oxidative damage is a significant factor in the pathogenesis of diabetes. In this study, to explore the role of Grx1 redox system in the pathogenesis of type 2 diabetes, clinical samples were collected, the levels of serum Grx1, oxidative stress, and glutathione were measured, and the correlation between inflammatory factors and diabetes was then analyzed.

Analysis of clinical indicators showed that the hyperglycemic patients had elevated concentrations of Grx1 in serum $(\mathrm{P}<0.05)$, in agreement with recently published data ( $\mathrm{Du}$ et al., 2014). However, the Total-SH concentrations were decreased $(\mathrm{P}<0.05$; Figure 1$)$. The analysis also revealed that the hyperglycemic patients had increased GST activity, reduced GSH levels, and elevated GSSG levels (Figure 2).

Genetics and Molecular Research 15 (3): gmr.15039000 
These results were indicative of elevated PSSG levels in the serum of hyperglycemic patients; this finding was consistent with numerous studies showing that PSSG levels in blood are elevated during active disease (Ghezzi, 2013). This meant that Grx1 and Grx1-regulated protein oxidative modification or glutathione modification may be the molecular markers of diabetes induced by oxidative stress and inflammation due to increased blood sugar. Currently, the data analysis on the correlation between the variables associated with diabetes and Grx1 is still in progress.
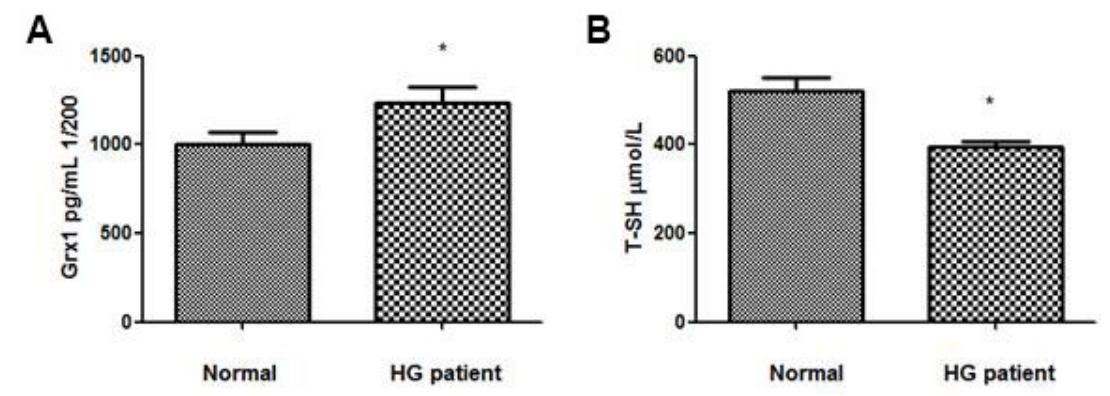

Figure 1. Assay for Grx1 and Total-SH concentrations in serum (normal group: 10 samples; HG patients: 50 samples). A. Grx1 concentration in serum. B. Total-SH concentration in serum. Bars represent mean \pm SEM. Significant differences $(\mathrm{P}<0.05)$ between normal and HG patients' data are indicated by an asterisk.

A

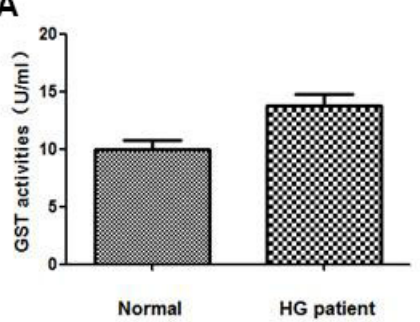

B

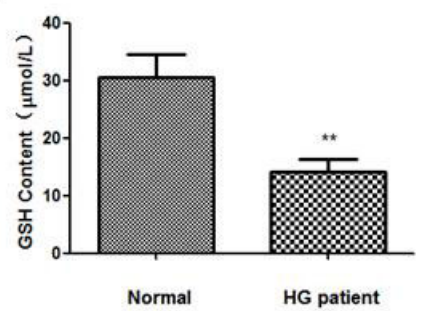

C

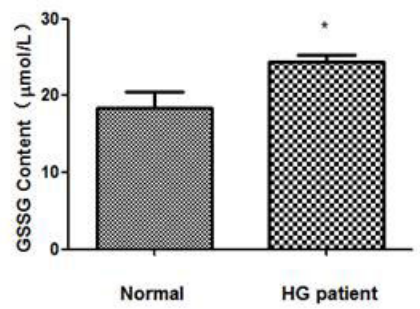

Figure 2. Analysis of the redox state of the body. A. GST activities. B. GSH content. C. GSSG content. Normal: a healthy person; HG patient: a patient with diabetes in the hyperglycemic state. Normals: 10 samples; HG patients: 50 samples. Bars represent mean \pm SEM. Significant differences $(* \mathrm{P}<0.05$ and $* * \mathrm{P}<0.01)$ between normals and HG patients are indicated by asterisks.

\section{Functions of the Grx1 redox system associated with the process of myocardial fibrosis in DCM rats}

Pathological changes associated with DCM include cardiac hypertrophy, interstitial fibrosis, and tissue necrosis. Myocardial fibrosis, including interstitial fibrosis and perivascular fibrosis, is a pathological feature of DCM (Asbun and Villarreal, 2006); therefore, the prevention and alleviation of myocardial fibrosis have become important goals in the treatment of DCM.

Hydroxyproline (Hyp) is an atypical amino acid in collagen, meaning that a collagen amount can be measured by determining the Hyp content. The quantification of Hyp content can accurately measure the overall level of collagen, which can be used to evaluate fibrotic 
lesions. To assess the effects of the myocardial Grx1 redox system of diabetic rats on the redox homeostasis of cardiac tissue, STZ and a high-fat diet were used to create a rat model of type 2 diabetes, which lasted for 12 weeks. Then, immunohistochemical analysis was used to detect the Grx1/GR protein expression levels in myocardial tissue, and an ELISA kit was used to determine the blood Grx1 level in the diabetic rats. Next, we employed the OxyBlot method to detect the oxidative damage to myocardial proteins, and commercial kits were utilized to evaluate the levels of oxidation using P-SH and Hyp, which works as an indicator of myocardial fibrosis. Finally, RT-PCR was applied to detection of the expression of inflammatory cytokines TNF- $\alpha$, IL-1B, and NF- $\kappa$ B p 65 . The results showed that the DCMrelated pathological changes were associated with significant oxidative damage (represented by the functional change of the Grx1 redox system and the increased protein carbonyl levels) and inflammation (increased expression of TNF- $\alpha$ and PAI-1).

Results of testing of oxidative stress levels in myocardial tissue and in serum showed that, according to the immunohistochemical data, the Grx1 levels in the left ventricular myocardium of the diabetic rats were significantly higher than in the control group, and the glutathione reductase (GR) levels in the diabetic rats were significantly lower than those in the control group ( $<<0.05$; Figure 3A). Radioimmunoassay results on blood Grx1 levels revealed that these levels in the diabetic rats were significantly increased ( $8.50 v s 6.37 ; \mathrm{P}<0.05$; Figure 3B), which was consistent with our results showing that Grx 1 tends to be upregulated in the plasma of hyperglycemic patients. OxyBlot results revealed that the protein carbonyl levels in myocardial tissue (reflecting protein oxidative damage) were significantly increased in the diabetic rats $(\mathrm{p}<0.01$; Figure $3 \mathrm{C})$. The above data indicated that Grx1 plays a significant role in the development of diabetes and cardiovascular complications of diabetes.

In this study, the results on myocardial Hyp and changes in the total amount of collagen indicated that the total myocardial collagen content in the diabetic rats was significantly increased 4 weeks after the onset of diabetes, and the total myocardial collagen content in the diabetic groups after 12 and 24 weeks were significantly higher than in the healthy groups, suggesting that the proliferation of collagen began to manifest itself at the early stage of the experimental diabetes. Results of myocardial fibrosis assays suggested that the total collagen content in the left ventricular myocardium of the diabetic group (D group, $\mathrm{n}=10$; Hyp $5.3 \pm 2.4$ $\mathrm{mg} / \mathrm{g}$ ) was significantly higher than that in the control group ( $\mathrm{N}$ group, $\mathrm{n}=10$; Hyp $2.8 \pm 0.9$ $\mathrm{mg} / \mathrm{g} ; \mathrm{P}<0.05$; Figure 4A). According to the relative quantitative RT-PCR results, the ratio of the gray values indicated that the expression levels of TNF- $\alpha$ and NF- $\kappa B$ in the rat myocardial tissue of the model group were significantly higher than those in the control group (Figure 4B).
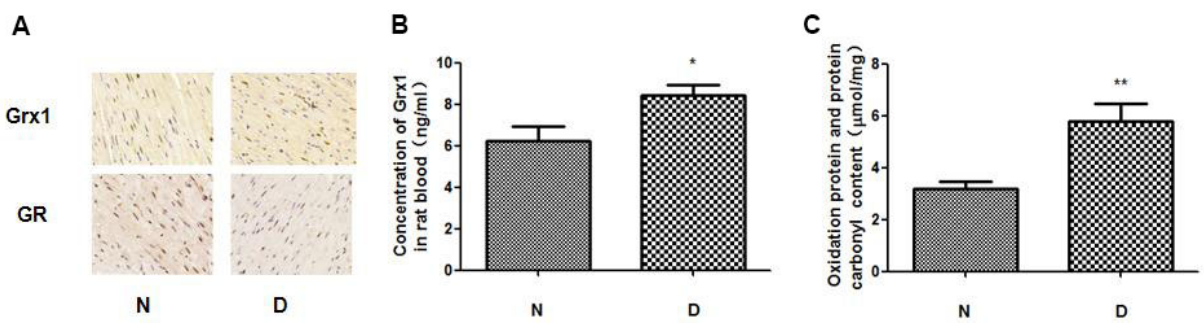

Figure 3. Characteristics of the oxidation-reduction system in diabetic rats (A) and dysfunction of the Grx1 system in diabetic rats (B). B. Concentration of Grx1 in rats' blood. C. The assay of oxidation-related proteins and protein carbonyl content. $\mathrm{N}=$ control group; $\mathrm{D}=$ diabetic group. Values are reported as means $\pm \mathrm{SD}$ of three measurements from separate experiments; $* \mathrm{P}<0.05 ; * * \mathrm{P}<0.01$ as compared with the control group.

Genetics and Molecular Research 15 (3): gmr.15039000 
A

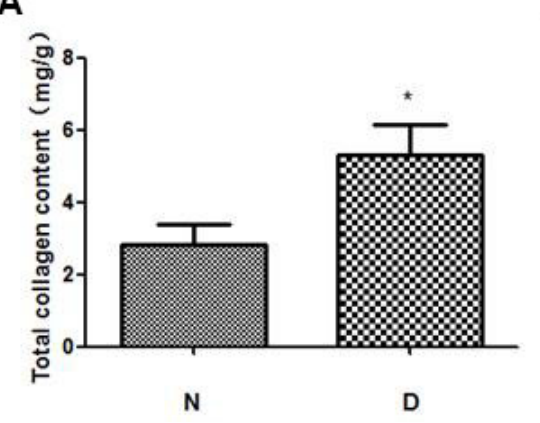

B

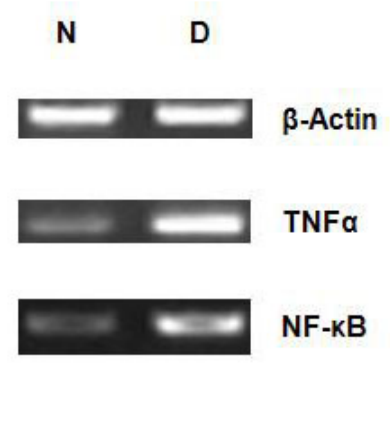

Figure 4. Pathological changes in diabetic rats. A. Assay of total collagen content. B. Expression levels of TNF- $\alpha$ and NF- $\kappa \mathrm{B}$ mRNA in rat myocardium tissue. $\mathrm{N}=$ control group; $\mathrm{D}=$ diabetic group. Values are reported as means $\pm \mathrm{SD}$ of three measurements from separate experiments; $* \mathrm{P}<0.05$ compared with the control group.

\section{Grx1 inhibited the expression of MMP-2 and MMP-9 in high-glucose-treated cardiac fibroblasts}

Diabetes can cause blood pressure changes, increased incidence of coronary heart disease, and aberrant cardiac structure and function. DCM is a specific cardiomyopathy and represents the end stage of diabetic myocardial disease (Resl et al., 2009). Studies in the early 1980s confirmed that diabetic patients can have myocardial fibrosis. In our previous study, it was also shown that autophagy protein expression and collagen content are significantly increased in the left ventricular myocardium of diabetic rats, thus clearly indicating fibrosis. The duration of hyperglycemia and the severity of myocardial fibrosis were found to correlate positively (Tarquini et al., 2011). By stimulating the overexpression of transforming growth factor (TGF), hyperglycemia and hyperinsulinemia promote the synthesis of ECM and deposition of fibrous tissue. Thus, by lowering the expression of TGF- $\beta 1$ and by reducing the synthesis of collagen, which is the main component of ECM, it is possible to inhibit myocardial fibrosis in DCM rats and to slow the progression of DMF (Gandhi et al., 2013).

Recently, Rajakumar et al. reported that high glucose levels can induce the expression of MMP-2 and MMP-9, which cause cardiac toxicity (Rajakumar et al., 2014). To explore the effects of inhibition of Grx1 on the profibrotic consequences of high-glucose-induced oxidative stress, western blotting was used to analyze the protein expression levels of MMP-2 and MMP-9. The results (Figure 5) showed that the expression levels of Grx1, MMP-2, and MMP-9 were all increased in cardiac fibroblasts in a time-dependent manner within 6 to 48 h. However, the increased protein expression levels of MMP-2 and MMP-9 induced by high glucose could be inhibited by Grx1, and this effect could be attenuated by the Grx1 inhibitor cadmium $\left(\mathrm{Cd}^{2+}\right)$. These results indicated that Grx1 can inhibit the process of myocardial fibrosis in DCM rats.

\section{Grx1 inhibits activation of the NF-кB signaling pathway in high-glucose-treated cardiac fibroblasts}

Studies have shown that upregulated ROS in myocardial cells, endothelial cells, and mesangial cells can promote the production of $\mathrm{NF}-\mathrm{\kappa B}$, which plays an important role in immune 

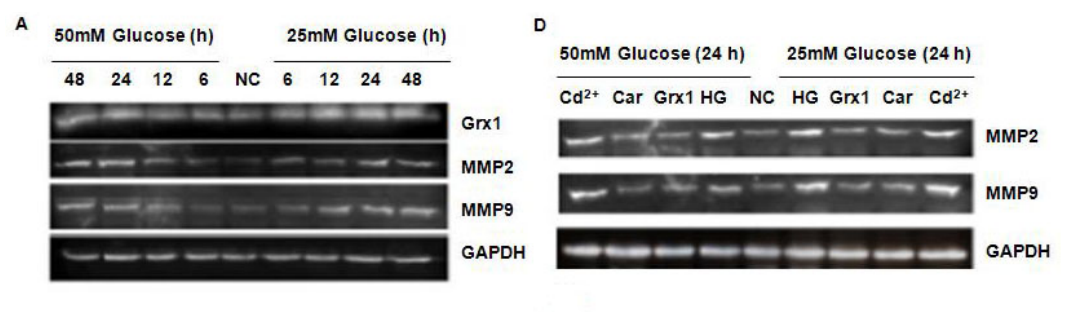

B
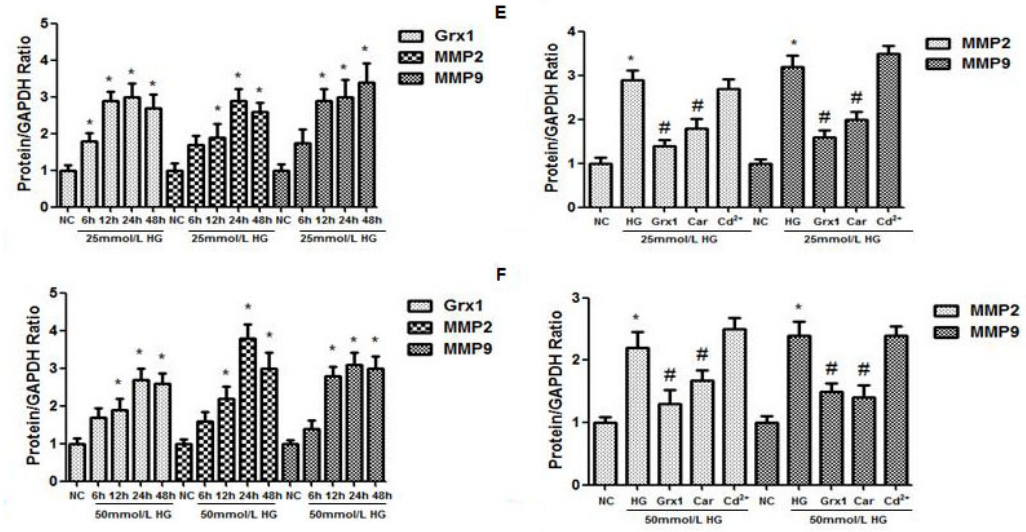

Figure 5. Expression levels of MMP-2 and MMP-9 in high-glucose-treated cardiac fibroblasts were analyzed by western blotting. A. The expression levels of Grx1, MMP-2, and MMP-9. Cardiac fibroblasts were incubated with or without 25 or $50 \mathrm{mmol} / \mathrm{L}$ D-glucose for $0,6,12,24$, or $48 \mathrm{~h}$. B. and C. Histogram analysis of the ratio. D. The expression levels of MMP-2 and MMP-9. Cardiac fibroblasts were incubated with or without 25 or $50 \mathrm{mmol} / \mathrm{L}$ D-glucose and with Grx1, Car, or $\mathrm{Cd}^{2+}$. E. and F. Histogram analysis of the ratio. NC: normal glucose $5.5 \mathrm{mM}, \mathrm{HG}$ : high glucose, $25 \mathrm{mM}, \mathrm{Cd}^{2+}$ : $\mathrm{CdCl}_{2} 10 \mu \mathrm{M}$, Grxl: glutaredoxin $15 \mu \mathrm{g} / \mathrm{mL}$, Car: carnosine $20 \mathrm{mM}$. Values are mean \pm $\mathrm{SD}$ of three measurements from separate experiments; ${ }^{*} \mathrm{P}<0.05$ compared with $\mathrm{NC}$; ${ }^{*} \mathrm{p}<0.05$ compared with HG.

and inflammatory responses and in apoptosis. Changes in the NF- $\kappa \mathrm{B}$ signaling pathway are associated with chronic disorders such as DMF and atherosclerosis (Lorenzo et al., 2011; Tsai et al., 2012; Kuo et al., 2013). NF-кB can be activated by external and internal factors, such as hyperglycemia, free fatty acids, ROS, TNF- $\alpha$, and some other proinflammatory factors. TNF- $\alpha$ expression is significantly increased in diabetic patients, and studies have shown its involvement in the development of DMF. Research has also confirmed that overexpression of inflammatory cytokines (TNF- $\alpha, \mathrm{IL}-1 \beta$ ) is not simply an epiphenomenon of DCM but is actively involved in the progression of diabetes-induced left ventricular dysfunction, and these expression levels significantly positively correlate with the severity of left ventricular dysfunction (Westermann et al., 2007a,b). TNF- $\alpha$ not only further amplifies inflammatory signals by inducing other inflammatory factors (thus aggravating the myocardial inflammation reactions) but also has a negative inotropic effect on myocardial cells in a concentrationdependent manner. The latter phenomenon participates in the pathological processes of myocardial fibrosis and apoptosis. These changes lead to myocardial remodeling and left ventricular dysfunction (Sun et al., 2007). To study the mechanism of the improvement of fibrosis by Grx 1 and to obtain evidence for possible medical treatment, we confirmed that the activation of NF- $\mathrm{kB}$ and the increased expression of its downstream inflammatory cytokine TNF- $\alpha$ can be inhibited at the mRNA level by exogenous Grx1 (Figure 6).

Genetics and Molecular Research 15 (3): gmr.15039000 
A

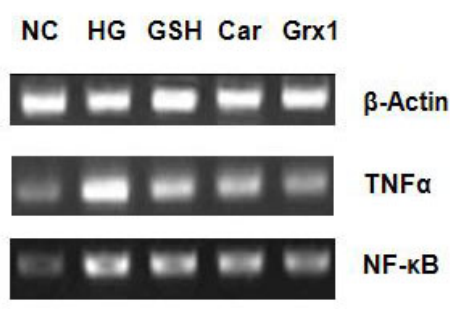

B

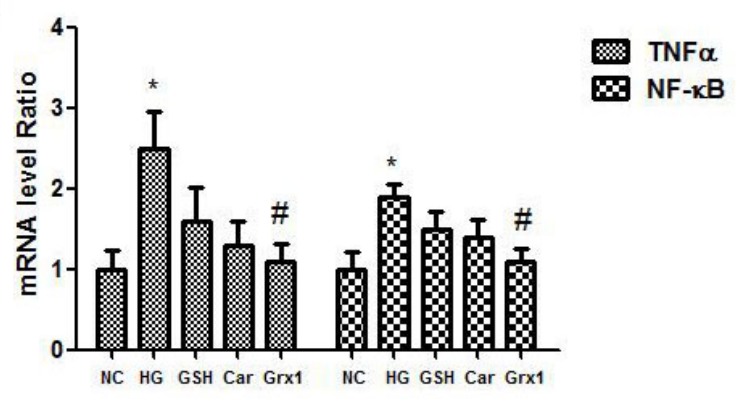

Figure 6. Effects of Grx1 on the expression levels of NF- $\kappa B$ and TNF- $\alpha$ mRNA in cardiac fibroblasts. A. RTPCR analysis of NF- $\kappa B$ and TNF- $\alpha$ mRNA expression after treatment with Grx1 under high-glucose conditions in cardiac fibroblasts. B. Histogram analysis of the expression of NF- $\kappa B$ and TNF- $\alpha$ mRNA after treatment with Grx1 under high-glucose conditions in cardiac fibroblasts; ${ }^{*} \mathrm{P}<0.05$ compared with $\mathrm{NC}$; $\mathrm{*} \mathrm{p}<0.05$ compared with HG. NC: normal glucose $5.5 \mathrm{mM}$, HG: high glucose $50 \mathrm{mM}$, GSH: GSH $10 \mu \mathrm{M}$, Car: carnosine $20 \mathrm{mM}$, Grx1: glutaredoxin $15 \mu \mathrm{g} / \mathrm{mL}$.

\section{DISCUSSION}

The incidence of diabetes has increased in recent years, and the reality of prevention and control is grim. An independent cardiac complication in diabetic patients, DCM is cardiac dysfunction in the absence of coronary artery disease and hypertension. Its incidence is high, and this pathology causes great harm. The pathogenesis of DCM is still elusive; it is thought to be related to a variety of factors including anomalies of glucose metabolism, lipid metabolism, and of energy metabolism in myocardial cells; myocardial fibrosis; oxidative stress; and cell death. Studies suggest that high blood glucose is a key initiating factor of DCM, which has the following two characteristics: glucose utilization problems in cardiac cells and damage to myocardial cells by the high blood glucose itself. Hyperglycemia has a direct toxic effect on myocardial cells and can induce production of large amounts of reactive oxygen and nitrogen species. Hyperglycemia can also stimulate the release of various cytokines and growth factors, thereby triggering myocardial oxidative stress and inflammation-related damage, which eventually leads to myocardial apoptosis and necrosis.

Long-term hyperglycemia causes a chronic, low-grade inflammatory state (Wen et al., 2013). The duration of hyperglycemia positively correlates with the severity of myocardial fibrosis (Tarquini et al., 2011). Myocardial hypertrophy and myocardial fibrosis can even occur in diabetic patients with mild hyperglycemia. Diabetes can induce production of ECM and the conversion imbalance of increased ECM synthesis and/or decreased ECM degradation, which causes excessive accumulation of ECM and can lead to myocardial fibrosis. Evidence shows that changes in ECM are associated with structural features of DCM (Yang et al., 2012) including increased levels of type I collagen and an increased ratio of type I/III collagen in DCM. Collagen degradation is a major cause of myocardial interstitial fibrosis in diabetes. Furthermore, cardiac MMP is a major regulator of matrix degradation, and increased activities of MMPs are associated with destruction and reconstruction of tissue architecture. The activities of pro-MMP-2 and MMP-2 are increased in DCM while MMP-9 activity shows only a tendency for an increase (Papazafiropoulou et al., 2010), which is also accompanied by an increase in MMP-1 activity. A number of studies have shown that the TNF- $\alpha$-induced 
activation of NF- $\mathrm{KB}$ and JNK/AP-1 signaling pathways and upregulation of MMP-9 expression can induce apoptosis in H9c2 cells and cardiovascular inflammation (Wu et al., 2013; Chen et al., 2013b; Yang et al., 2013). In a diabetic state, elevated levels of cytokines (such as TGF- $\beta 1$, TNF- $\alpha$ ) and accumulation of free radicals of oxygen can promote the activation of MMPs. Excessive activation of MMPs often causes incomplete tissue degradation, which promotes migration of fibroblasts and fibrosis; these phenomena may be closely related to collagen metabolism disorders and heart failure associated with DCM (Yang et al., 2010).

A recent study showed that NF- $\kappa \mathrm{B}$ pathways are involved in the development and progression of myocardial fibrosis (Mariappan et al., 2010). NF- $\kappa B$ is involved in the development of DCM by causing inflammation, oxidative stress, endothelial dysfunction, apoptosis, and myocardial fibrosis. In cultured cardiac fibroblasts, inhibition of NF- $\kappa \mathrm{B}$ can suppress the proliferation of fibroblasts, thereby decreasing myocardial fibrosis (Wu et al., 2009). Nonetheless, there are no targeted NF- $\kappa B$-inhibitors (drugs); thus, the development of NF-kB-blocking drugs with high selectivity will likely become the new goal for the treatment of DCM. On the basis of the important role of NF- $\kappa B$ in the pathophysiology of DCM, the exogenous regulation of NF- $\kappa B$ activation may be an effective strategy against DCM.

Our analysis of clinical indicators revealed that in the serum of hyperglycemic patients, Grx1 concentration was elevated $(\mathrm{P}<0.05)$, while total-SH content was reduced $(\mathrm{P}<0.05)$. GST activity was increased, GSH content was decreased, and GSSG levels were increased, indicating that Grx1 and Grx1-regulated protein oxidative and glutathione modifications may serve as molecular markers of diabetes induced by oxidative stress and inflammation due to increased blood sugar. Animal studies have shown that in diabetic models, blood glucose, triglycerides, cholesterol, and LDL are all significantly elevated; insulin levels are significantly reduced; and insulin resistance becomes evident. There are also functional changes in the Grx 1 system of left ventricular myocardial tissues in diabetic rats, in which the Grx1 levels were upregulated and GR and P-SH levels were downregulated in left ventricular myocardial tissue and blood. In myocardial cells, activated (reduced) Grx depends on GR, which is thought to be due to the inhibition of GR function and weakened functioning of the Grx system in diabetic rats. Because P-SH levels are reduced and the protein carbonyl groups, which reflect protein oxidative damage, are significantly upregulated, the weakened functioning of the Grx 1 redox system is believed to be linked to enhanced protein oxidation. In addition, it was found that total collagen content of the myocardium in diabetic rats was significantly increased 1 month after the onset of experimental diabetes, indicating that the Grx1 antioxidant system is involved in the development and progression of DMF.

Our previous study (Li et al., 2014) revealed that exogenous Grx1 can alleviate oxidative stress and apoptosis in porcine coronary endothelial cells by activating the eNOS/ NO system and inhibiting the activation of JNK/NF- $\kappa B$ signaling pathways. This approach can also attenuate H9c2 cell apoptosis (induced by high glucose) by inhibiting the activation of caspase 8- or 3-mediated apoptotic pathways and JNK/c-Jun signaling pathways (Zhang et al., 2014b). Nevertheless, there are few reports on the impact of Grx1 on myocardial fibrosis in high-glucose environments. Therefore, from the standpoint of the internal relations among signal pathways (and rules that govern them) and the factors related to myocardium remodeling in diabetic rats, we used RT-PCR and western blotting to measure the mRNA and protein levels of TNF- $\alpha$ and MMP-2/9. In addition, zymography was used to analyze the activity of MMP-9. These data provided a new theoretical basis for identification of 
Grx1's mechanism of action. This study seems to be the first to show that endogenous Grx1 is highly expressed in cardiac fibroblasts exposed to a high glucose concentration, and that exogenous Grx1 can significantly inhibit the expression of MMP-2 and MMP-9 in cardiac fibroblasts exposed to high glucose. These data suggest that the Grx1 antioxidant system is involved in the development and progression of DMF and has inhibitory effects on DMF. Additionally, it was found that the mechanism of Grx1-driven suppression of myocardial fibrosis (induced by high glucose) is related to the inhibition of NF- $\mathrm{BB} / \mathrm{TNF}-\alpha$ activation. This finding is consistent with the results of another research group (Gallogly et al., 2010), who demonstrated that lowered Grx1 levels after siRNA transfection can reduce NF- $\mathrm{kB}$ genedependent transcription activity, and furthermore, to decrease the mRNA and protein levels of Bcl-2 and Bcl-xL, which are antiapoptotic target genes of NF- $\mathrm{KB}$, thus increasing apoptosis of cardiac H9c2 cells. Moreover, our study showed that high-glucose-induced upregulation of TNF- $\alpha$ and MMP-9 mRNA, as well as the activity of MMP-9, can be inhibited by Grx1. These data are in agreement with a recent study involving high-glucose-triggered cardiac toxicity induced by the expression of MMP-2 and MMP-9 (Rajakumar et al., 2014). Recent studies confirm that antioxidants antagonize high-glucose-induced myocardial apoptosis by inhibiting the activation of oxidative-stress-stimulated JNK/NF- $\mathrm{KB}$ signaling pathways (Kuo et al., 2013). Our previous study also revealed that the mechanism - via which antioxidant carnosine attenuates high-glucose-induced myocardial cell apoptosis - is related to the inhibited expression of p-JNK/pc-Jun (Qi et al., 2014). Therefore, on the basis of existing lines of evidence, we can hypothesize that the Grx1 system not only inhibits the profibrotic action of oxidative stress but may also inhibit the profibrotic effects of NF- $\kappa \mathrm{B}$ and inflammatory cytokines (such as TNF- $\alpha$ and IL-1 $\beta$ ).

S-glutathionylation of proteins is an important means to regulate intracellular redox balance. As a sulfhydryl-disulfide bond oxidoreductase - by catalyzing the reduction of protein disulfide bonds, mixed disulfide bonds, and of glutathione protein (Pr-S-S-G) - the Grx 1 enzyme regulates post-translational modifications of thiol-containing proteins including those in JNK/c-Jun signaling pathways (Allen and Mieyal, 2012; Bansal et al., 2013; Chen et al., 2013a; Lu and Holmgren, 2014). One group recently reported that Grx1-mediated uncoupling of glutathione from IKK $\beta$ Cys-179 plays a key role in diabetic complications by regulating the autocrine and paracrine actions of proinflammatory cytokines (Shelton et al., 2009). Grx1-mediated coupling of glutathione to NF- $\mathrm{KB}$ was found to perform a protective function in a vascular anti-inflammatory mechanism (Liao et al., 2010). In a transgenic mouse model of chronic myocardial infarction (Adluri et al., 2012), it was found that the overexpression of human Grx1 can increase the phosphorylation level of Akt and the DNA-binding activity of NF- $\mathrm{BB}$, which promote angiogenesis and reduce ventricular remodeling, oxidative stress, and myocardial apoptosis. Therefore, diabetic cardiac fibrosis is determined by the interaction and balance among a variety of factors including oxidative stress. The specific mechanism and effects of the Grx1 redox system on the processes of DMF need to be studied further, including whether this mechanism is related to Grx1mediated S-glutathionylation and whether synergy exists between the Grx1/GSH system and other endogenous antioxidants such as carnosine.

\section{Conflicts of interest}

The authors declare no conflict of interest.

Genetics and Molecular Research 15 (3): gmr.15039000 


\section{ACKNOWLEDGMENTS}

The authors sincerely thank the many people who assisted with the study and manuscript. Research supported by grants from the Committee of Education Science Foundation of Heilongjiang Province (\#12541902 and \#12541905); the Foundation of Qiqihar Science and Technology Bureau (\#SFGG-201331 and \#SFGG-201455); and the Key Project of the University Students' Innovation and Pioneering Training (\#201411230012).

\section{REFERENCES}

Adluri RS, Thirunavukkarasu M, Zhan L, Dunna NR, et al. (2012). Glutaredoxin-1 overexpression enhances neovascularization and diminishes ventricular remodeling in chronic myocardial infarction. PLoS One 7: e34790 http://dx.doi.org/10.1371/journal.pone.0034790.

Allen EM and Mieyal JJ (2012). Protein-thiol oxidation and cell death: regulatory role of glutaredoxins. Antioxid. Redox Signal. 17: 1748-1763 http://dx.doi.org/10.1089/ars.2012.4644.

Aragno M, Mastrocola R, Alloatti G, Vercellinatto I, et al. (2008). Oxidative stress triggers cardiac fibrosis in the heart of diabetic rats. Endocrinology 149: 380-388 http://dx.doi.org/10.1210/en.2007-0877.

Asbun J and Villarreal FJ (2006). The pathogenesis of myocardial fibrosis in the setting of diabetic cardiomyopathy. J. Am. Coll. Cardiol. 47: 693-700 http://dx.doi.org/10.1016/j.jacc.2005.09.050.

Bansal G, Das D, Hsieh CY, Wang YH, et al. (2013). IL-22 activates oxidant signaling in pulmonary vascular smooth muscle cells. Cell. Signal. 25: 2727-2733 http://dx.doi.org/10.1016/j.cellsig.2013.09.001.

Chen CA, De Pascali F, Basye A, Hemann C, et al. (2013a). Redox modulation of endothelial nitric oxide synthase by glutaredoxin-1 through reversible oxidative post-translational modification. Biochemistry 52: 6712-6723 http:// dx.doi.org/10.1021/bi400404s.

Chen Y, Decker KF, Zheng D, Matkovich SJ, et al. (2013b). A nucleus-targeted alternately spliced Nix/Bnip3L protein isoform modifies nuclear factor $\mathrm{\kappa B}(\mathrm{NF \kappa B})$-mediated cardiac transcription. J. Biol. Chem. 288: 15455-15465 http:// dx.doi.org/10.1074/jbc.M113.452342.

De Benedetto ML, Capo CR, Ferri A, Valle C, et al. (2014). Glutaredoxin 1 is a major player in copper metabolism in neuroblastoma cells. Biochim. Biophys. Acta 1840: 255-261 http://dx.doi.org/10.1016/j.bbagen.2013.09.008.

Du Y, Zhang H, Montano S, Hegestam J, et al. (2014). Plasma glutaredoxin activity in healthy subjects and patients with abnormal glucose levels or overt type 2 diabetes. Acta Diabetol. 51: 225-232 http://dx.doi.org/10.1007/s00592-013$\underline{0498-2 .}$.

Ernande L and Derumeaux G (2012). Diabetic cardiomyopathy: myth or reality? Arch. Cardiovasc. Dis. 105: 218-225 http://dx.doi.org/10.1016/j.acvd.2011.11.007.

Gallogly MM, Shelton MD, Qanungo S, Pai HV, et al. (2010). Glutaredoxin regulates apoptosis in cardiomyocytes via NFkappaB targets Bcl-2 and Bcl-xL: implications for cardiac aging. Antioxid. Redox Signal. 12: 1339-1353 http:// dx.doi.org/10.1089/ars.2009.2791.

Gandhi S, Srinivasan B and Akarte AS (2013). Aliskiren improves insulin resistance and ameliorates diabetic renal vascular complications in STZ-induced diabetic rats. J. Renin Angiotensin Aldosterone Syst. 14: 3-13 http://dx.doi. org $/ 10.1177 / 1470320312452766$.

Ghezzi P (2013). Protein glutathionylation in health and disease. Biochim. Biophys. Acta 1830: 3165-3172 http://dx.doi. org/10.1016/j.bbagen.2013.02.009.

Jellis C, Wright J, Kennedy D, Sacre J, et al. (2011). Association of imaging markers of myocardial fibrosis with metabolic and functional disturbances in early diabetic cardiomyopathy. Circ Cardiovasc Imaging 4: 693-702 http://dx.doi. org/10.1161/CIRCIMAGING.111.963587.

Kuo WW, Wang WJ, Tsai CY, Way CL, et al. (2013). Diallyl trisufide (DATS) suppresses high glucose-induced cardiomyocyte apoptosis by inhibiting JNK/NFkB signaling via attenuating ROS generation. Int. J. Cardiol. 168: 270-280 http://dx.doi.org/10.1016/j.ijcard.2012.09.080.

Lekli I, Mukherjee S, Ray D, Gurusamy N, et al. (2010). Functional recovery of diabetic mouse hearts by glutaredoxin-1 gene therapy: role of Akt-FoxO-signaling network. Gene Ther. 17: 478-485 http://dx.doi.org/10.1038/gt.2010.9.

Li CJ, Lv L, Li H and Yu DM (2012). Cardiac fibrosis and dysfunction in experimental diabetic cardiomyopathy are ameliorated by alpha-lipoic acid. Cardiovasc. Diabetol. 11: 73 http://dx.doi.org/10.1186/1475-2840-11-73.

Li S, Sun Y, Qi X, Shi Y, et al. (2014). Protective effect and mechanism of glutaredoxin 1 on coronary arteries endothelial

Genetics and Molecular Research 15 (3): gmr.15039000 
cells damage induced by high glucose. Biomed. Mater. Eng. 24: 3897-3903 10.1016/j.bbagen.2013.02.009.

Liao BC, Hsieh CW, Lin YC and Wung BS (2010). The glutaredoxin/glutathione system modulates NF-kappaB activity by glutathionylation of p65 in cinnamaldehyde-treated endothelial cells. Toxicol. Sci. 116: 151-163 http://dx.doi. org $/ 10.1093 /$ toxsci/kfq098.

Lorenzo O, Picatoste B, Ares-Carrasco S, Ramírez E, et al. (2011). Potential role of nuclear factor $\kappa \mathrm{B}$ in diabetic cardiomyopathy. Mediators Inflamm. 2011: $652097 \mathrm{http} / / \mathrm{dx} . d o i . o r g / 10.1155 / 2011 / 652097$.

Lu J and Holmgren A (2014). The thioredoxin antioxidant system. Free Radic. Biol. Med. 66: 75-87 http://dx.doi. org/10.1016/j.freeradbiomed.2013.07.036.

Mariappan N, Elks CM, Sriramula S, Guggilam A, et al. (2010). NF-kappaB-induced oxidative stress contributes to mitochondrial and cardiac dysfunction in type II diabetes. Cardiovasc. Res. 85: 473-483 http://dx.doi.org/10.1093/ $\underline{\mathrm{cvr} / \mathrm{cvp} 305 .}$.

Marine K, Meehan P, Lyons AC and Curley MA (2013). Inequity of patient assignments: fact or fiction? Crit. Care Nurse 33: 74-77 http://dx.doi.org/10.4037/ccn2013399.

Miki T, Yuda S, Kouzu H and Miura T (2013). Diabetic cardiomyopathy: pathophysiology and clinical features. Heart Fail. Rev. 18: 149-166 http://dx.doi.org/10.1007/s10741-012-9313-3.

Papazafiropoulou A, Perrea D, Moyssakis I, Kokkinos A, et al. (2010). Plasma levels of MMP-2, MMP-9 and TIMP-1 are not associated with arterial stiffness in subjects with type 2 diabetes mellitus. J. Diabetes Complications 24: 20-27 http://dx.doi.org/10.1016/j.jdiacomp.2008.10.004.

Qi XD, Zhang CJ, Shi Y, Yu HT, et al. (2014). Carnosine inhibits cardiomyocyte apoptosis via modulation of the JNK and NF-kappaB signaling cascade in H9c2 cells exposed to high glucose. Chin. J. Biochem. Mol. Biol. 30: 156-162.

Rajakumar P, Anandhan R, Vadla GP and Vellaichamy E (2014). Synthesis and cardio protective biological applications of glucodendrimers by H9C2 cell studies. Carbohydr. Polym. 99: 403-414 http://dx.doi.org/10.1016/j. carbpol.2013.08.040.

Resl M, Hülsmann M, Pacher R and Clodi M (2009). [Heart failure in diabetes]. Wien. Med. Wochenschr. 159: 134-140 http://dx.doi.org/10.1007/s10354-009-0645-0.

Shelton MD, Distler AM, Kern TS and Mieyal JJ (2009). Glutaredoxin regulates autocrine and paracrine proinflammatory responses in retinal glial (muller) cells. J. Biol. Chem. 284: 4760-4766 http://dx.doi.org/10.1074/jbc.M805464200.

Sun M, Chen M, Dawood F, Zurawska U, et al. (2007). Tumor necrosis factor-alpha mediates cardiac remodeling and ventricular dysfunction after pressure overload state. Circulation. 115: 1398-1407. http://dx.doi.org/10.1161/ CIRCULATI ONAHA.106.643585

Tarquini R, Lazzeri C, Pala L, Rotella CM, et al. (2011). The diabetic cardiomyopathy. Acta Diabetol. 48: 173-181 http:// dx.doi.org/10.1007/s00592-010-0180-x.

Tsai KH, Wang WJ, Lin CW, Pai P, et al. (2012). NADPH oxidase-derived superoxide anion-induced apoptosis is mediated via the JNK-dependent activation of NF- $\mathrm{KB}$ in cardiomyocytes exposed to high glucose. J. Cell. Physiol. 227: 1347-1357 http://dx.doi.org/10.1002/jcp.22847.

Wen HL, Liang ZS, Zhang R and Yang K (2013). Anti-inflammatory effects of triptolide improve left ventricular function in a rat model of diabetic cardiomyopathy. Cardiovasc. Diabetol. 12: 50 http://dx.doi.org/10.1186/1475-2840-12-50.

Westermann D, Van Linthout S, Dhayat S, Dhayat N, et al. (2007a). Cardioprotective and anti-inflammatory effects of interleukin converting enzyme inhibition in experimental diabetic cardiomyopathy. Diabetes 56: 1834-1841 http:// dx.doi.org/10.2337/db06-1662.

Westermann D, Van Linthout S, Dhayat S, Dhayat N, et al. (2007b). Tumor necrosis factor-alpha antagonism protects from myocardial inflammation and fibrosis in experimental diabetic cardiomyopathy. Basic Res. Cardiol. 102: 500$507 \mathrm{http}: / / \mathrm{dx}$.doi.org/10.1007/s00395-007-0673-0.

Wu HT, Sie SS, Kuan TC and Lin CS (2013). Identifying the regulative role of NF- $\kappa B$ binding sites within promoter region of human matrix metalloproteinase 9 (mmp-9) by TNF- $\alpha$ induction. Appl. Biochem. Biotechnol. 169: 438-449 http://dx.doi.org/10.1007/s12010-012-9958-3.

Wu M, Han M, Li J, Xu X, et al. (2009). 17beta-estradiol inhibits angiotensin II-induced cardiac myofibroblast differentiation. Eur. J. Pharmacol. 616: 155-159 http://dx.doi.org/10.1016/j.ejphar.2009.05.016.

Yang B, Li M, Shi ZG and Feng QZ (2012). Bosentan ameliorates the expression of fibrotic related growth factors and collagen-1 in diabetic mice. Anadolu Kardiyol. Derg. 12: 621-627 10.5152/akd.2012.213.

Yang CM, Lee IT, Lin CC, Wang CH, et al. (2013). c-Src-dependent MAPKs/AP-1 activation is involved in TNF- $\alpha$ induced matrix metalloproteinase-9 expression in rat heart-derived H9c2 cells. Biochem. Pharmacol. 85: 1115-1123 http://dx.doi.org/10.1016/j.bcp.2013.01.013.

Yang J, Fan XH, Guan YQ, Li Y, et al. (2010). MMP-2 gene polymorphisms in type 2 diabetes mellitus diabetic retinopathy. Int. J. Ophthalmol. 3: 137-140 10.3980/j.issn.2222-3959.

Genetics and Molecular Research 15 (3): gmr.15039000 
Zhang CJ, Hou JC, Qi XD, Wang B, et al. (2014a). Glutaredoxin 1 Regulates High Glucose-induced Autophagy in Cardiomyocytes. Chin. J. Biochem. Mol. Biol. 30: 912-918.

Zhang CJ, Hou JC, Wang B, Qi XD, et al. (2014b). Glutaredoxin 1 Suppresses the Apoptotic Signal Pathway of Caspase-8/3 and JNK /c-Jun in H9c2 Cell Induced by High Glucose. Chin. J. Biochem. Mol. Biol. 30: 579-583. 